LBL-PUB-42768, January, 1999

\title{
Exclusive Vector Meson Production in Relativistic Heavy Ion Collisions
}

\author{
Spencer R. Klein and Joakim Nystrand \\ Lawrence Berkeley National Laboratory, Berkeley, CA 94720
}

\begin{abstract}
Exclusive vector meson production reactions such as $\mathrm{Au}+\mathrm{Au} \rightarrow \mathrm{Au}+$ $\mathrm{Au}+\mathrm{V}$, where $V=\rho, \omega, \phi$ or $J / \psi$ can proceed through photon-Pomeron and photon-meson interactions. Photons from the electromagnetic field of one nucleus interact coherently with the other nucleus. Photonuclear cross sections are scaled from $\gamma p$ data, and convoluted with the photon spectrum to find the exclusive rates.

The cross sections at the RHIC and LHC heavy ion colliders are huge, $10 \%$ of the total hadronic cross section at RHIC, and $50 \%$ at LHC. These accelerators may be useful as vector meson factories. With iodine beams at RHIC, $640 \rho$ are produced each second (10 10 year); with calcium at the LHC the rate is $240 \mathrm{kHz}$. The $\phi$ rates are $39 \mathrm{~Hz}$ at RHIC and $15 \mathrm{kHz}$ at LHC, while the $J / \psi$ rate is $0.3 \mathrm{~Hz}$ at RHIC and $780 \mathrm{~Hz}$ at the LHC.

Because of the coherent couplings, the reactions kinematics are similar to coherent two-photon interactions; we discuss the interplay between the two reactions.
\end{abstract}

(Submitted to Physical Review C.)

Typeset using REVTEX 


\section{INTRODUCTION}

The electromagnetic fields of ultrarelativistic heavy ions may be treated as fields of virtual photons. When two nuclei pass by at small impact parameters, the photon field of one nucleus can produce a photonuclear interaction in the other nucleus. Most studies of photonuclear reactions in heavy ion collisions have considered hard interactions, such as heavy quark production [1] or photonuclear breakup [2].

We consider here the exclusive reaction $A+A \rightarrow A+A+V$, where $A$ is a heavy nucleus, and $V$ is a vector meson. This reaction can proceed via photon-Pomeron or photon-meson interactions, where the photon comes from the electromagnetic field of one nucleus, and the Pomeron or meson couples to the other nucleus. At sufficiently high energies, the momentum transfers from the nuclei are small enough that both couplings are coherent to the entire nucleus. Then, the nucleus remains intact, or nearly intact. We calculate the production rates and rapidity distributions for the $\rho^{0}, \omega, \phi$ and $\mathrm{J} / \psi$.

These reactions will be studied experimentally at the upcoming Relativistic Heavy Ion Collider (RHIC) [3] now under construction at Brookhaven National Laboratory [4, and the Large Hadron Collider (LHC) [5] being built at CERN. For later reference, Table [ gives the expected luminosities for various beam species at these two colliders.

\section{PHOTON FLUX AND PHOTONUCLEAR CROSS SECTIONS}

The calculations are simplest in the rest frame of one of the nuclei, labelled the target nucleus. The total production is found by taking each nucleus as the target in turn. This calculation divides into two parts: determination of the photonuclear interaction cross section, and calculation of the effective photon flux from the emitting nucleus.

In this frame, the photon energy $k$ is large, and the momentum transfer squared from the target nucleus is small. The momentum transfer $t$ has longitudinal $t_{\|}$and transverse components $t_{\perp}$, with $t=t_{\|}+t_{\perp}$. Since the initial state longitudinal momentum is small, $t_{\perp}$ is the final state momentum transverse to the photon direction. When $t_{\perp}=0,|t|=\left|t_{\text {min }}\right|=$ $\sqrt{M_{v}^{2} / 4 k}$.

The coherent coupling of the photons and Pomerons limits their virtuality to $1 / R_{A}$, where $R_{A}$ is the nuclear radius, or about $30 \mathrm{MeV} /$ c for heavy nuclei like gold or lead. This virtuality is small enough that it can be neglected here, with the photon effectively real in the target frame.

The photon flux from a relativistic heavy nucleus is given by the Weizsäcker-Williams approach. Here $\gamma=E_{A} / m_{A} \gg 1$, where $E_{A}$ and $m_{A}$ are the energy and mass of the nucleus. The photon flux at a distance $r$ from the nucleus is

$$
\frac{d^{3} N_{\gamma}(k, r)}{d k d^{2} r}=\frac{Z^{2} \alpha x^{2}}{\pi^{2} k r^{2}} K_{1}^{2}(x)
$$

where $k$ is the photon energy, $Z$ is the nuclear charge, $K_{1}$ a modified Bessel function with $x=k r / \gamma$. The target frame $\gamma$ corresponds to $\gamma=2 \Gamma^{2}-1$, where $\Gamma$ is the Lorentz boost in the center of mass frame (laboratory frame for collider geometry). Here, and throughout the paper, we take $\hbar=c=1$. 
In an exclusive interaction, there can be no accompanying hadronic interactions. In a hard sphere model, this occurs if the impact parameter $b>2 R_{A}$, where $R_{A}=1.2 A^{1 / 3}$ $\mathrm{fm}$. We use a more accurate approach, calculating, the probability of one or more hadronic interactions as a function of impact parameter. The nuclear density for a nucleus $A$ a distance $s$ from its center is modelled with a Woods- Saxon distribution for symmetric nuclei

$$
\rho_{A}(s)=\frac{\rho_{0}}{1+\exp \left[\left(s-R_{W S}\right) / d\right]}
$$

where the radius $R_{W S}$ and skin depth $d$ are based on fits to electron scattering data [6] and $\rho^{0}$ is fixed by the normalization. This electron scattering data probes the proton distribution in the nucleus. If the neutron are differently distributed from the protons, this could affect the cross section..

The interaction probability at a given impact parameter is related to the overlap function

$$
T_{A A}(|\vec{b}|)=\int d^{2} \vec{r} T_{A}(\vec{r}) T_{A}(\vec{r}-\vec{b})
$$

where $\vec{r}$ and $\vec{b}$ are 2-dimensional vectors perpendicular to the direction of travel $z$. The nuclear thickness function is

$$
T_{A}(\vec{r})=\int d z \rho_{A}\left(\sqrt{|\vec{r}|^{2}+z^{2}}\right)
$$

The number of nucleon-nucleon collisions follows a Poisson distribution with mean $T_{A A}(b) \sigma_{N N}$, with $\sigma_{N N}$ the total nucleon-nucleon interaction cross section. The probability of having no hadronic interactions is then

$$
P_{0 H}(b)=\exp \left(-T_{A A}(b) \sigma_{N N}\right) .
$$

We use $\sigma_{N N}=52 \mathrm{mb}$ at a beam energy of $100 \mathrm{GeV}$, rising to $88 \mathrm{mb}$ at $2.9 \mathrm{TeV}$ [7].

We assume that the range of the Pomeron or meson field is much smaller than the size of the nucleus [8]. For $b<2 R_{A}$, a form factor can be required to accurately model the electromagnetic fields inside the emitting nucleus; we neglect this tiny correction.

The total photon flux is given by Eq. (1) integrated over all $r$, modulated by the noninteraction probability.

$$
\frac{d N_{\gamma}(k)}{d k}=\int_{0}^{\infty} 2 \pi b d b P_{0 H}(b) \int_{0}^{R} \frac{r d r}{\pi R_{A}^{2}} \int_{0}^{2 \pi} d \phi \frac{d^{3} N_{\gamma}(k, b+r \cos (\phi))}{d k d^{2} r}
$$

The $b$ integral runs over impact parameter and $r$ and $\phi$ over the surface of the target nucleus, for a given $b$. This process averages the field strength over the nucleus, neglecting the variation with $r$. Since the vector meson production is coherent over the entire nucleus, this averaging is appropriate. The integral is evaluated numerically; the result is shown in Fig. 1. This flux is approximately equal to the photon flux in the region $r>2 R_{A}$, which can be found analytically:

$$
\frac{d N_{\gamma}(k)}{d k}=\frac{2 Z^{2} \alpha}{\pi k}\left(X K_{0}(X) K_{1}(X)-\frac{X^{2}}{2}\left(K_{1}^{2}(X)-K_{0}^{2}(X)\right)\right)
$$


where $X=2 R_{A} k / \gamma$. This approximation is shown by the dotted line in Fig. 1.

The $\gamma A \rightarrow V A$ cross sections are found with a Glauber calculation, using the observed $\gamma p \rightarrow V p$ cross sections as input. Data on $\gamma p \rightarrow V p$ covers a wide energy range, from low energy fixed target photon beams [9] to ep collisions at HERA [10]. These cross sections may be parameterized as

$$
\left.\frac{d \sigma(\gamma p \rightarrow V p)}{d t}\right|_{t=0}=b_{V}\left(X W^{\epsilon}+Y W^{-\eta}\right)
$$

where $W$ is the center of mass energy, and Table II gives the constants $b_{V}, X, Y, \epsilon$ and $\eta$, which are determined from fits to data [11]. The $X, \epsilon$ term represents the Pomeron exchange portion of the cross section. It rises slowly with energy $(\epsilon \sim 0.22)$. This slow rise has been attributed to the increasing size and density of the proton; the increasing $W$ probes smaller proton momentum fractions $x$. The $Y, \eta$ term, is for meson exchange, primarily the $f_{0}$ [12]. The meson exchange term falls rapidly as $W$ rises. For the $\phi$ and $J / \psi$, meson exchange is heavily suppressed, and the reaction occurs only through Pomeron exchange. For the $J / \psi$, $\epsilon \gg 0.22$; the rapid rise has been ascribed to either a breakdown of the soft Pomeron model or to threshold effects [10]. Because of this behavior, the $J / \psi$ calculations must be treated with caution, especially at center of mass energies beyond that probed by HERA.

To relate this to nuclei, we make use of the optical theorem and an Eikonalization technique. Following vector dominance [9],

$$
\left.\frac{d \sigma(\gamma p \rightarrow V p)}{d t}\right|_{t=0}=\left.\frac{4 \pi \alpha}{f_{v}^{2}} \frac{d \sigma(V p \rightarrow V p)}{d t}\right|_{t=0}
$$

with a similar relationship true for nuclei. Here, $\mathrm{t}$ is the squared 4-momentum transfer between the proton and vector meson, $\alpha$ is the electromagnetic coupling constant, $e^{2} / \hbar c$, and $f_{v}$ is the vector meson-photon coupling,

$$
\frac{f_{v}^{2}}{4 \pi}=\frac{M_{v} \alpha^{2}}{3 \Gamma_{V \rightarrow e^{+} e^{-}}}
$$

with $M_{v}$ the vector meson mass and $\Gamma_{V \rightarrow e^{+} e^{-}}$the leptonic decay partial width. However, measurements of $f_{v}$ from leptonic decay widths disagree with photoproduction data [13]. A correction is required to account for non-diagonal coupling through higher mass vector mesons, as implemented in the generalized vector dominance model (GVDM). For the $\rho$, GVDM increases $f_{v}^{2}$ by $20 \%$ [13]. Because $f_{v}^{2}$ is used twice, the error largely cancels here. Values for $f_{v}^{2} / 4 \pi$ are given in Table [II.

Using the optical theorem, the total cross section is

$$
\sigma_{\text {tot }}^{2}(V p)=\left.16 \pi \frac{d \sigma(V p \rightarrow V p)}{d t}\right|_{t=0} .
$$

At $W=10 \mathrm{GeV}$, we find $24 \mathrm{mb}, 26 \mathrm{mb}, 12 \mathrm{mb}$, and $1.0 \mathrm{mb}$ for the $\rho, \omega, \phi$, and $J / \psi$ total cross sections respectively. These values are consistent with theoretical and experimental expectations [9]. The scattering cross section from heavy nuclei can be found by a Glauber calculation

$$
\sigma_{t o t}(V A)=\int d^{2} \vec{r}\left(1-e^{-\sigma_{t o t}(V p) T_{A A}(\vec{r})}\right)
$$


The optical theorem for nucleus $A$ and GVDM are then used to find

$$
\left.\frac{d \sigma(\gamma A \rightarrow V A)}{d t}\right|_{t=0}=\frac{\alpha \sigma_{t o t}^{2}(V A)}{4 f_{v}^{2}}
$$

These $d \sigma /\left.d t\right|_{t=0}$ are shown in Fig. 3 for gold nuclei. Also shown are the results of scaling the $\gamma p$ cross sections by $A^{2}$ and $A^{4 / 3}$ [3]. The $\rho, \omega$, and $\phi$ results vary only slowly with energy. The minimum around photon-proton center of mass energy $W_{\gamma p}=10 \mathrm{GeV}$ for the $\rho$ and $\omega$ marks the transition from meson dominated to Pomeron dominated reactions. $\phi$ production, which is mediated only by Pomerons, shows a monotonic energy dependence.

For the light mesons, $d \sigma /\left.d t\right|_{t=0}$ is almost independent of energy, flatter than the corresponding proton functions, which appears unchanged in the $A^{2}$ and $A^{4 / 3}$ scaling curves. In contrast, $d \sigma /\left.d t\right|_{t=0}$ for the $J / \psi$ rises rapidly with energy. Part of the difference is the different $\gamma p$ cross section energy dependence, and part is the smaller $J / \psi$ interaction cross section. Because $\sigma_{\rho N}$ is large, $\rho$ interact near the nuclear surface, and $\sigma_{\rho A}$ is the geometric cross section $\pi R_{A}^{2}$ which is almost independent of $k$. However, $\sigma_{J / \psi N}$ is much smaller, and the $J / \psi$ interactions occur throughout the nucleus. As long as $\sigma_{J / \psi N} R_{A} \rho_{0} \ll 1$, the rising $\sigma_{J / \psi N}$ will reflect itself in $\sigma_{J / \psi A}$. These cases correspond to the black disk and weak absorption limits respectively.

The HERA results which provide the high energy $\gamma p$ input data extend up to about $W_{\gamma p}$ $=180 \mathrm{GeV}(k=16 \mathrm{TeV}$ in the target frame), with higher energy points extrapolations. Although the HERA data covers the entire RHIC energy range, the extrapolations are required for LHC. The uncertainty is especially problematic for the $J / \psi$, where $d \sigma /\left.d t\right|_{t=0}$ must eventually flatten out, to avoid dominating the total photonuclear cross section.

The total cross section depends on the slope of $d \sigma / d t$. For proton targets, the cross section can be parameterized as $d \sigma / d t=A_{v} \exp \left(-b t+c|t|^{2}\right)$ [14]. The slope $b$, and derivative $c$ represent the sum of the proton size and the range of the interaction. Here, for simplicity we take $c=0$ with little loss of accuracy. Nuclei are much larger than protons, so $b$ is dominated by the nuclear size, with $b \sim R^{2}$ and $d \sigma / d t$ is dominated by the nuclear form factor. This is important because experimental study of these exclusive reactions depends on their small $t_{\perp}$ [15 which is determined by the form factor.

The form factor is the Fourier transform of the nuclear density profile. Unfortunately, the Woods-Saxon distribution does not have an analytic form factor. Instead, for simplicity we approximate the Woods-Saxon distribution as a hard sphere, with radius $R_{A}$, convoluted with a Yukawa potential with range $r=0.7 \mathrm{fm}$ [16]. The Fourier transform of this convolution is the product of the two individual transforms:

$$
F(q=\sqrt{|t|})=\frac{4 \pi \rho_{0}}{A q^{3}}\left[\sin \left(q R_{a}\right)-q R_{a} \cos \left(q R_{A}\right)\right]\left[\frac{1}{1+a^{2} q^{2}}\right]
$$

Figure 2 compares Eq. (14) with the numerical transform of the Woods-Saxon distribution for gold. The agreement is excellent.

In addition to coherent production, there is also incoherent vector meson production, where the photon interacts with a single nucleon in the target. Incoherent interactions have a larger average $t$, and occur at a somewhat lower rate. They will not be further considered here, but they will be an essential component of any experimental analysis.

The photonuclear cross section is 


$$
\sigma(\gamma A \rightarrow V A)=\left.\frac{d \sigma(\gamma A \rightarrow V A)}{d t}\right|_{t=0} \int_{t_{\min }}^{\infty} d t|F(t)|^{2}
$$

For narrow resonances, $t_{\min }=\left(M_{v}^{2} / 4 k\right)^{2}$. Because of its width, the $\rho$ is more complicated, and the cross section must be calculated using a Breit-Wigner resonance:

$$
\frac{d \sigma}{d M_{\pi \pi}}=\frac{2}{\pi} \frac{\sigma_{0} \Gamma_{\rho} M_{\rho} M_{\pi \pi}}{\left(M_{\pi \pi}^{2}-M_{\rho}^{2}\right)^{2}+\Gamma_{\rho}^{2} M_{\rho}^{2}} .
$$

Here, $M_{\rho}$ is the pole position of the resonance, $\sigma_{0}$ the total cross section (neglecting phase space corrections) and $M_{\pi \pi}$ the final state invariant mass. The Breit-Wigner width $\Gamma_{\rho}$ includes the phase space correction, with $\Gamma_{\rho}=\Gamma_{0}\left(p_{\pi} / p_{0}\right)^{3}\left(M_{\rho} / M_{\pi \pi}\right)$ [17] where $p_{\pi}$ is the decay pion momentum in the $\rho$ rest frame, with $p_{0}=358 \mathrm{MeV} / \mathrm{c}$ the pion momentum for $M_{\pi \pi}=M_{\rho}$ and $\Gamma_{0}$ is the pole $\rho$ width. The observed resonance shape is the convolution of this Breit-Wigner with the photon spectrum. Figure 0 compares the resulting spectrum with the input Breit-Wigner. With the Breit-Wigner, the cross section is about $5 \%$ lower than for a narrow resonance with the same coupling. The phase space correction naturally cuts off the $M_{\pi \pi}$ spectrum at $2 m_{\pi}$; we add an upper limit $M_{\pi \pi}<M_{\rho}+5 \Gamma_{0}$, matching the HERA analysis from which the $\gamma p$ parameters are extracted [14]. The correction for higher masses is a few percent.

Most studies of $\gamma p \rightarrow \rho p$ have modelled the exclusive $\pi^{+} \pi^{-}$spectrum with a Breit-Wigner $\rho$ plus a non-resonant $\pi^{+} \pi^{-}$term, and an interference term [18]:

$$
\frac{d \sigma}{d M_{\pi \pi}}=\left|\frac{A \sqrt{M_{\pi \pi} M_{\rho} \Gamma_{\rho}}}{M_{\pi \pi}^{2}-M_{\rho}^{2}+i M_{\rho} \Gamma_{\rho}}+B\right|^{2} .
$$

The ZEUS collaboration found $A=-2.75 \pm 0.04 \mu \mathrm{b}^{1 / 2}$ and $B=1.84 \pm 0.06(\mu \mathrm{b} / \mathrm{GeV})^{1 / 2}$ [14]. With the interference term, $d \sigma / d M_{\pi \pi}$ falls rapidly at large $W$, and a correction for $M_{\pi \pi}>M_{\rho}+5 \Gamma_{0}$ is negligible.

As nuclear size increases, the non-resonant term becomes less important. At lower energies, for heavy nuclei it is at most a few percent. For large nuclei, the $k$ dependence of $B$ is unknwon, so we neglect non-resonant production. For the nuclei considered, this should be less than a $10 \%$ effect on the $\pi^{+} \pi^{-}$rate.

The photonuclear cross sections are shown in Figure 4 . The total cross sections follow a similar pattern to $d \sigma /\left.d t\right|_{t=0}$. At low energies, the nuclear form factor intrudes, and eliminates coherent production, with the cutoff energy depending on the nuclear radius and the final state mass. The $\omega$ and $\phi$ curves cross because the $\omega$ includes a meson contribution that decreases with increasing energy, while the $\phi$ does not and because $\sigma_{\phi N}<\sigma_{\omega N}$, with the exact relationship varying slightly with energy.

Coherent photonuclear interactions are not a new concept. Several groups studied them experimentally and theoretically in the 1960s and 1970's, using real photon beams. More recent experiments, using virtual photons from muon scattering are less comparable because of the significant (compared with $1 / R_{A}$ ) photon virtuality.

Most of the earlier calculations were used to extract parameters such as $\sigma_{V N}$ and $R_{A}$ from data. Our calculations are broadly similar. However, most of the earlier calculations used a disk model of the nucleus, with $t_{\perp}$ determined by a 2-dimensional Fourier transform 
of a black disk, and $t_{\|}$contributing only to a phase shift. Many of these calculations also included a real part for the forward scattering amplitude. For the $\rho$, the real part is 20$30 \%$ of the imaginary part, producing a 5\% correction to the total amplitude [9]. For other mesons, the phases should be comparable (when meson exchange is present) or smaller, for Pomeron-only interactions. In all cases, the correction should decrease slowly at higher energies.

The experimental results can also test our calculations. Because of the complications due to differing (often poorly described) treatments of backgrounds, $\rho$ width and the nonresonant $\pi^{+} \pi^{-}$, we limit our comparisons to narrow resonances. Even for narrow resonances, there are complications. Few experiments cover the complete $t$ range; extrapolations are required to find the cross sections. We compare results using $d \sigma /\left.d t\right|_{t=0}$, because less extrapolation is required.

An experiment at Cornell studied $\omega$ production from photons with average energy 8.2 $\mathrm{GeV}$ striking a copper target, and found $d \sigma /\left.d t\right|_{t=0}=9.6 \pm 1.2 \mathrm{mb} / \mathrm{GeV}^{2}$ [19. This compares well with our calculated $d \sigma /\left.d t\right|_{t=0}=9.5 \mathrm{mb} / \mathrm{GeV}^{2}$ for that system. Another group studied $\phi$ production from $8.3 \mathrm{GeV}$ photons incident on copper and lead targets, and found $d \sigma /\left.d t\right|_{t=0}=$ $4.1 \pm 0.7$ and $19 \pm 3 \mathrm{mb} / \mathrm{GeV}^{2}$ respectively [20]. We predict higher cross sections, $d \sigma /\left.d t\right|_{t=0}=$ 5.4 and $38 \mathrm{mb} / \mathrm{GeV}^{2}$ respectively. However, with the values of $f_{\phi}$ and $\sigma_{\phi N}$ used in the original analysis, we find $d \sigma /\left.d t\right|_{t=0}=3.9$ and $26 \mathrm{mb} / \mathrm{GeV}^{2}$ respectively, in agreement with the data. The discrepancy may stem from the large correction(60\% for copper, $140 \%$ for lead) to cover the full angular acceptance. This correction is based on the optical model, and so depends on $f_{\phi}$ and $\sigma_{\phi N}$. Still, these comparisons provide a useful check of our methods.

\section{HEAVY ION CROSS SECTIONS}

By integrating the photonuclear cross section over the photon spectrum, the total cross section is found:

$\sigma(A A \rightarrow A A V)=\int d k \frac{d N_{\gamma}(k)}{d k} \sigma(\gamma A \rightarrow V A)=\left.\int_{0}^{\infty} d k \frac{d N_{\gamma}(k)}{d k} \int_{t_{\min }}^{\infty} d t \frac{d \sigma(\gamma A \rightarrow V A)}{d t}\right|_{t=0}|F(t)|^{2}$

where the photon flux is from Eq. (6). The total cross sections for vector meson production at RHIC and LHC for a variety of beam species are shown in Table [II. The corresponding production rates are shown in Table $\mathbb{I V}$. These rates are very high, with exclusive meson cross sections about $10 \%$ of the total hadronic cross section for gold at RHIC, and $50 \%$ for lead at LHC. If the hadronic interaction model, Eq. (5), is replaced with a hard sphere cutoff, $\left(b>2 R_{A}\right)$, the rates rise about $5 \%$.

Previously, we considered a model where $\gamma A$ cross sections were scaled from $\gamma p$ treating the nucleus as a black disk, with $\sigma \sim A^{2 / 3}$. In that model, the photonuclear cross sections had a different $k$ dependence, shown by the open triangles in Fig. 3. This scaling predicted cross sections much lower than are found here [21]. Most other studies of photon-Pomeron interactions have neglected the coherent Pomeron-nucleus coupling. Baur, Hencken and Trautman found $\sigma(A u p \rightarrow A u p V)=3.5 \mathrm{mb}$ using the Weizsäcker-Williams photon flux for gold incident on a free proton target at RHIC [22]. 
The final state rapidity is determined by the ratio of the photon energy and $t_{\|}$in the lab frame:

$$
y=\frac{1}{2} \ln \frac{k}{\sqrt{\left|t_{\min }\right|}}=\ln \frac{k}{2 M_{V}}
$$

so $d \sigma / d y=k d \sigma / d k$ and $d \sigma / d y$ is found by a change of variables. For the $\rho$, the cross section must be integrated over $M_{\pi \pi} \cdot d N / d y$ is shown in Fig. 6, for the $\rho$ (Breit-Wigner), $\phi$ and $J / \psi$, for production with gold at RHIC and calcium at LHC. The cross section is largest at low photon energies, corresponding to $y<0$, because the photon flux drops as $k$ rises. The peaking is larger for the $\rho$ and $\omega$, where meson exchange increases the cross section at low energies.

At RHIC, when photons from both nuclei are added together, separate peaks appear in $d N / d y$. At LHC, the higher energies spread the distribution over a much wider rapidity range, and the double peaked structure largely disappears, except for the $\rho$.

The total perpendicular momentum spectrum is dominated by the two nuclear form factors, through the cutoff on the emitted photon $p_{\perp}$ and through the slope $b$ of $d \sigma / d t$. The (quadrature) sum of these is roughly $\sqrt{2} \hbar / R_{A}$, or about $45 \mathrm{MeV} / \mathrm{c}$ for gold. This cutoff is a clear signature of coherent interactions, shared with $\gamma \gamma$ and coherent double Pomeron interactions. Because of the exclusive production and small $p_{\perp}$, these events can be easily separated from background such as grazing nuclear collisions, beam gas interactions and incoherent photonuclear interactions [23].

Other authors have considered coherent double-Pomeron interactions in heavy ion collisions [24]. If reactions where the nuclei collide are excluded, the cross section is very small, and depends critically on the range of the Pomeron [8] 25].

\section{MULTIPLE VECTOR MESON PRODUCTION}

Because the cross sections are so large, the probability of having multiple interactions in a single nucleus-nucleus encounter is nonnegligible. This can be quantified by considering the meson production probability $P(V)=d \sigma / 2 \pi b d b$ for a single collision. Figure 7 shows this probability for various mesons for (a) gold beams at RHIC and (b) lead beams at LHC; the probabilities reach the $1 \%$ level. For the $\rho$, we show results from two different hadronic interactions models: the Woods-Saxon model, Eq. (5), and also a hard sphere $b>2 R_{A}$ result. Although the two models predict very similar overall rates, the probability is significantly affected around $b=2 R_{A}$, doubling the maximum probability at LHC.

This probability is high enough that, even in the absence of correlations [26], significant numbers of vector meson pairs should be produced. Neglecting correlations, the pair production probability is $P(V)^{2} / 2$ for identical pairs and $P\left(V_{1}\right) P\left(V_{2}\right)$ for nonidentical pairs. These cross section and corresponding yearly rates are tabulated in Table $\square$.

Triples and higher multiples are also possible. Of course, final state interactions may affect what is observed in a detector. 


\section{INTERPLAY BETWEEN PHOTONUCLEAR AND TWO-PHOTON INTERACTIONS}

Two-photon physics is expected to be a significant activity at RHIC [3] [15] and LHC [22] [27. Because the kinematics for both photonuclear interactions and two-photon collisions is dominated by the coherent coupling to the two nuclei, and consequently by the nuclear form factors, the reactions have similar kinematics. So, the specific pathway may not be determinable from the final state.

The rates for photonuclear interactions are considerably higher than for comparable two-photon reactions. For example, the $\phi$ production rate with gold beams at RHIC is about $8 \mathrm{~Hz}$. For comparison, the rates for $\gamma \gamma \rightarrow \eta^{\prime}$ and $\gamma \gamma \rightarrow f_{0}(980)$ [21] are 0.13 and $0.02 \mathrm{~Hz}$ respectively. At higher masses, the situation is similar, and photonuclear vector meson production may overshadow two-photon production of scalar and tensor final states, complicating meson spectroscopy. It is worth noting that the photonuclear $\rho \rho$ rate is many times larger than that expected from $\gamma \gamma$ production [27]. On the other hand, the same techniques developed to study $\gamma \gamma$ physics at heavy ion colliders are effective at selecting photonuclear interactions.

The similarity of the two interactions can lead to some interesting opportunities. For example, when the final states are indistinguishable, interference can occur between the photonuclear and $\gamma \gamma$ production channels. Interference between leptonic decays of photonuclear produced vector mesons and Bethe-Heitler $e^{+} e^{-}$pairs has been studied previously [12]; at future heavy ion colliders, many more channels will be accessible.

Despite the similarity, the different $d N / d y$ spectra may allow statistical separation of $\gamma \gamma$ and photonuclear interactions. This is because the photon flux scales as $1 / k$, while the Pomeron/meson couplings have a much weaker energy dependence. The $d N / d y$ distribution for photonuclear production is broader than for two-photon production. This is especially true for photon-meson interactions, where the $1 / k$ photon spectrum combines with a cross section that drops with energy to form two peaks at large positive and negative rapidity, corresponding to low energy photons from the two nuclei in turn. Figure VI compares the two distributions for $\gamma \gamma \rightarrow f_{2}$ (1270) and for production of a hypothetical vector meson with the same mass. Two vector meson models are shown: one based on the $\omega$ couplings and another with only Pomeron coupling. The two are very similar at $y=0$, but the $\omega$ model peaks about $10 \%$ higher.

It may also be possible to separate the two classes by their different impact parameter dependence. A two-photon interaction can occur at a significant distance from both nuclei, while a photonuclear interaction must occur inside or very near a nucleus. So, the two classes will have a different impact parameter dependence. In a collision, photon exchange can leave the nuclei excited in a giant dipole resonance, regardless of other activity; the excitation probability depends on the impact parameter [22]. This may allow us to statistically separate the two classes.

In a broader study, it will also be possible to separate the two classes by measuring how the rates vary with $A$; the meson and Pomeron flux rises should be less $A$ dependent than photons. This study will require data with a variety of different beam species. 


\section{CONCLUSIONS}

We have calculated the rates and rapidity distribution for exclusive photonuclear production of vector mesons in heavy ion collisions, using a Glauber model calculation. The LHC results involve photons with energies considerably above those currently available. Although this adds to the physics interest, it also introduces some uncertainty into the rates presented

here. For the $J / \psi$, in particular, the spectrum must soften at some photon energy, and the rates given here may be overestimates.

The production rates are large enough that heavy ion colliders could be used as vector meson factories. The $\phi$ and $J / \psi$ production rates at LHC are comparable to those at existing or planned meson factories based on $e^{+} e^{-}$annihilation.

These rates are higher than comparable two-photon or double-Pomeron interactions. Thus, these photonuclear interactions should be the dominant source of exclusive interactions at heavy ion colliders. In addition to single interactions, multiple vector meson production from the same ion pair should be measurable.

We thank Jørgen Randrup for suggesting the hard sphere plus Yukawa potential form factor. We would like to acknowledge useful conversations with Stan Brodsky and Ralph Engel. This work was supported by the US DOE, under contract DE-AC-03-76SF00098. 


\section{REFERENCES}

[1] M. Greiner et al., Phys. Rev. C51, 911 (1995).

[2] N. Baron and G. Baur, Phys. Rev. C48, 1999 (1993).

[3] J. Nystrand and S. Klein, nucl-ex/9811007, Nov., 1998, in Proc. Workshop on Photon Interactions and the Photon Structure, eds. G. Jarlskog and T. Sjöstrand, Lund, Sweden, Sept., 1998.

[4] Conceptual Design for the Relativistic Heavy Ion Collider, BNL-52195, May, 1989, Brookhaven National Laboratory.

[5] N. Ahmad et al.(the ALICE Collaboration), CERN/LHCC 95-71/LHCC/P3, Dec., 1995.

[6] R. C. Barrett and D. F. Jackson, Nuclear Sizes and Structure, Oxford University Press, 1977.

[7] C. Caso et al.(Particle Data Group), Eur. Phys. J. C3, 1 (1998).

[8] B. Müller and A. J. Schramm, Nucl. Phys. A523, 677 (1991).

[9] T. H. Bauer et al., Rev. Mod. Phys. 50, 261 (1978).

[10] N. Cartiglia, hep-ph/9703245, Mar., 1997.

[11] J. A. Crittenden, Exclusive Production of Neutral Vector Mesons at the Electron-Proton Collider HERA, Springer-Verlag, 1997.

[12] D. Leith in Electromagnetic Interactions of Hadrons, ed. A. Donnachie and G. Shaw, Plenum Press, 1978, and references therein.

[13] A. Pautz and G. Shaw, Phys. Rev. C57, 2648 (1998).

[14] J. Breitweig et al., Eur. Phys. J 2, 247 (1998).

[15] S. Klein and E. Scannapieco, hep-ph/9806358, in Photon '97, ed. A. Buijs and F. C. Erne, World Scientific, 1997.

[16] K. T. R. Davies and J. R. Nix, Phys. Rev. C14, 1977 (1976).

[17] J. D. Jackson, Il. Nuovo Cimento, 34, 1644 (1964).

[18] P. Söding, Phys. Lett. 19, 702 (1966).

[19] H. J. Behrend et al., Phys. Rev. Lett. 24, 1246 (1970); J. Abramson et al., Phys. Rev. Lett. 36, 1428 (1976).

[20] G. McClellan et al., Phys. Rev. Lett. 26, 1593 (1971).

[21] S. Klein and J. Nystrand, STAR Note 347, June, 1998. Available on the web at http://www.rhic.bnl.gov/star/starlib/doc/www/sno/ice/sn0347.html.

[22] G. Baur, K. Hencken and D. Trautman, J. Phys. G 24, 1657 (1998).

[23] J. Nystrand and S. Klein, hep-ex/9711021, in Hadron Spectroscopy, ed. S. Chung and H. Willutzki, AIP Conf. Proc. 432, 1998.

[24] R. Engel et al., Z. Phys. C74, 687 (1997).

[25] A. J. Schramm and D. H. Reeves, Phys. Rev. D55, 7312 (1997).

[26] S. Klein and J. Nystrand, in preparation.

[27] E. Lippma, et al.(the FELIX collaboration), preprint CERN/LHCC- 97/45, 1997.

[28] D. Brandt, K. Eggert and A. Morsch, CERN AT/94-05, LHC Note 264, March, 1994 (unpublished). 


\section{TABLES}

TABLE I. Luminosity and beam kinetic energy for several heavy ion beams at RHIC and LHC. Because RHIC will be dedicated to heavy ion acceleration, it is likely to run a wider variety of beams than LHC. The RHIC luminosities are from Ref. [4. Different references quote somewhat different heavy ion luminosity for LHC; these numbers are calculated from Table 3 of Ref. [28], assuming 2 experiments, and that (as suggested) average luminosity is $45 \%$ of peak luminosity.

\begin{tabular}{lrrr}
\hline \hline Particle & Machine & Max Beam Energy & Design Luminosity \\
\hline Gold & RHIC & $100 \mathrm{GeV} / \mathrm{n}$ & $2 \times 10^{26} \mathrm{~cm}^{-2} s^{-1}$ \\
Iodine & RHIC & $104 \mathrm{GeV} / \mathrm{n}$ & $2.7 \times 10^{27} \mathrm{~cm}^{-2} s^{-1}$ \\
Silicon & RHIC & $125 \mathrm{GeV} / \mathrm{n}$ & $4.4 \times 10^{28} \mathrm{~cm}^{-2} s^{-1}$ \\
Lead & LHC & $2.76 \mathrm{TeV} / \mathrm{n}$ & $1 \times 10^{26} \mathrm{~cm}^{-2} s^{-1}$ \\
Calcium & LHC & $3.5 \mathrm{TeV} / \mathrm{n}$ & $2 \times 10^{30} \mathrm{~cm}^{-2} s^{-1}$ \\
\hline \hline
\end{tabular}

TABLE II. Constants for $\gamma p \rightarrow V p$ production. The slopes $b_{V}$ are in $\mathrm{GeV}^{-2}$, while $X$ and $Y$ are in $\mu$ barns, for $W$ in $\mathrm{GeV}$ [11. The $f_{v}$ values are from Eq. (10).

\begin{tabular}{lrrrrrr}
\hline \hline Meson & $b_{V}$ & $X$ & $\epsilon$ & $Y$ & $\eta$ & $f_{v}^{2} / 4 \pi$ \\
\hline$\rho^{0}$ & 11 & 5.0 & 0.22 & 26.0 & 1.23 & 2.02 \\
$\omega$ & 10 & 0.55 & 0.22 & 18.0 & 1.92 & 23.1 \\
$\phi$ & 7 & 0.34 & 0.22 & & & 13.7 \\
$J / \psi$ & 4 & 0.0015 & 0.80 & & & 10.4 \\
\hline \hline
\end{tabular}

TABLE III. Cross sections, in millibarns, for production of vector mesons for the beams given in Table 2.

\begin{tabular}{lrrrrr}
\hline \hline Meson & RHIC-Au & RHIC-I & RHIC-Si & LHC-Pb & LHC-Ca \\
\hline$\rho^{0}$ & 590 & 230 & 8.4 & 5200 & 120 \\
$\omega$ & 59 & 24 & 0.9 & 490 & 12 \\
$\phi$ & 39 & 14 & 0.4 & 460 & 7.6 \\
$\mathrm{~J} / \psi$ & 0.29 & 0.11 & 0.0036 & 32 & 0.39 \\
\hline \hline
\end{tabular}

TABLE IV. Meson production rates, in Hz, at design luminosity for various beams.

\begin{tabular}{lrrrrr}
\hline \hline Meson & (RHIC-Au $)$ & RHIC-I & RHIC-Si & LHC-Pb & LHC-Ca \\
\hline$\rho^{0}$ & 120 & 620 & 370 & 520 & 230,000 \\
$\omega$ & 12 & 63 & 42 & 49 & 23,000 \\
$\phi$ & 7.9 & 39 & 18 & 46 & 15,000 \\
$\mathrm{~J} / \psi$ & 0.058 & 0.30 & 0.16 & 3.2 & 780 \\
\hline \hline
\end{tabular}


TABLE V. Cross sections and rates for production of vector mesons pairs in gold beams at RHIC and lead beams at LHC. The yearly rates are for $10^{7}$ second/year at RHIC and $10^{6}$ second/year of operation at LHC. The LHC $\rho J / \psi$ rates should be used with caution because of the energy extrapolation.

\begin{tabular}{lrrrr}
\hline \hline Meson & $\sigma(\mu \mathrm{b})($ RHIC-Au $)$ & Rate/year $($ RHIC-Au $)$ & $\sigma(\mu \mathrm{b})(\mathrm{LHC}-\mathrm{Pb})$ & Rate/year $(\mathrm{LHC}-\mathrm{Pb})$ \\
\hline$\rho^{0} \rho^{0}$ & 720 & $1,400,000$ & 8,800 & 880,000 \\
$\omega \omega$ & 6.2 & 12,000 & 73 & 7,300 \\
$\phi \phi$ & 3.8 & 7,500 & 76 & 7,600 \\
$\rho^{0} \omega$ & 133 & 270,000 & 1,600 & 160,000 \\
$\rho^{0} \phi$ & 104 & 210,000 & 1,640 & 160,000 \\
$\omega \phi$ & 9.6 & 19,000 & 150 & 15,000 \\
$\rho^{0} J / \psi$ & 1.3 & 2,700 & 200 & 20,000 \\
\hline \hline
\end{tabular}




\section{FIGURES}

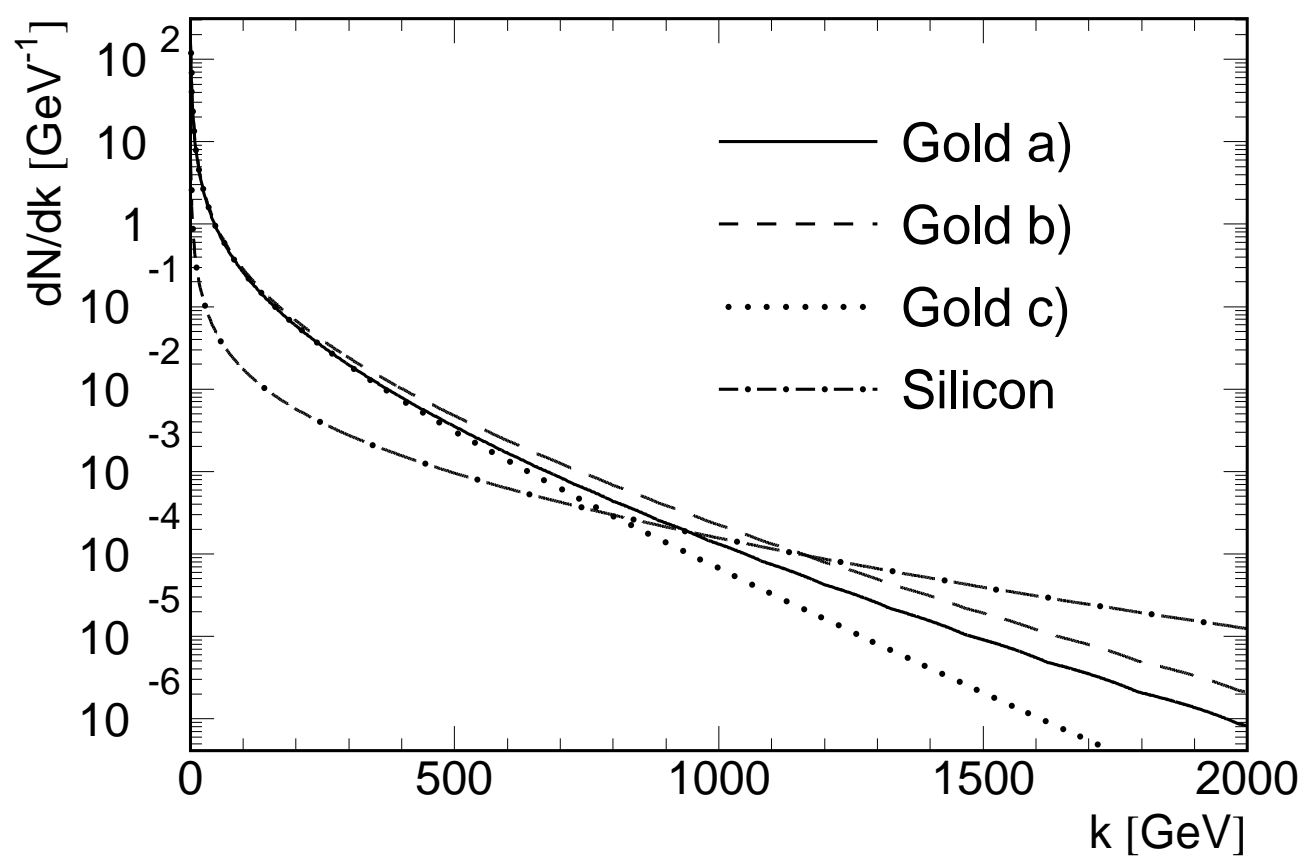

FIG. 1. The photon flux for gold beams using (a) the hadronic interaction probability described in the text, (b) the hard sphere approximation, (c), the photon flux for $b>2 R_{A}$, Eq. (7), and for silicon beams with the same approach as in (a). Both are at RHIC energies, with $k$ in the target frame.. 


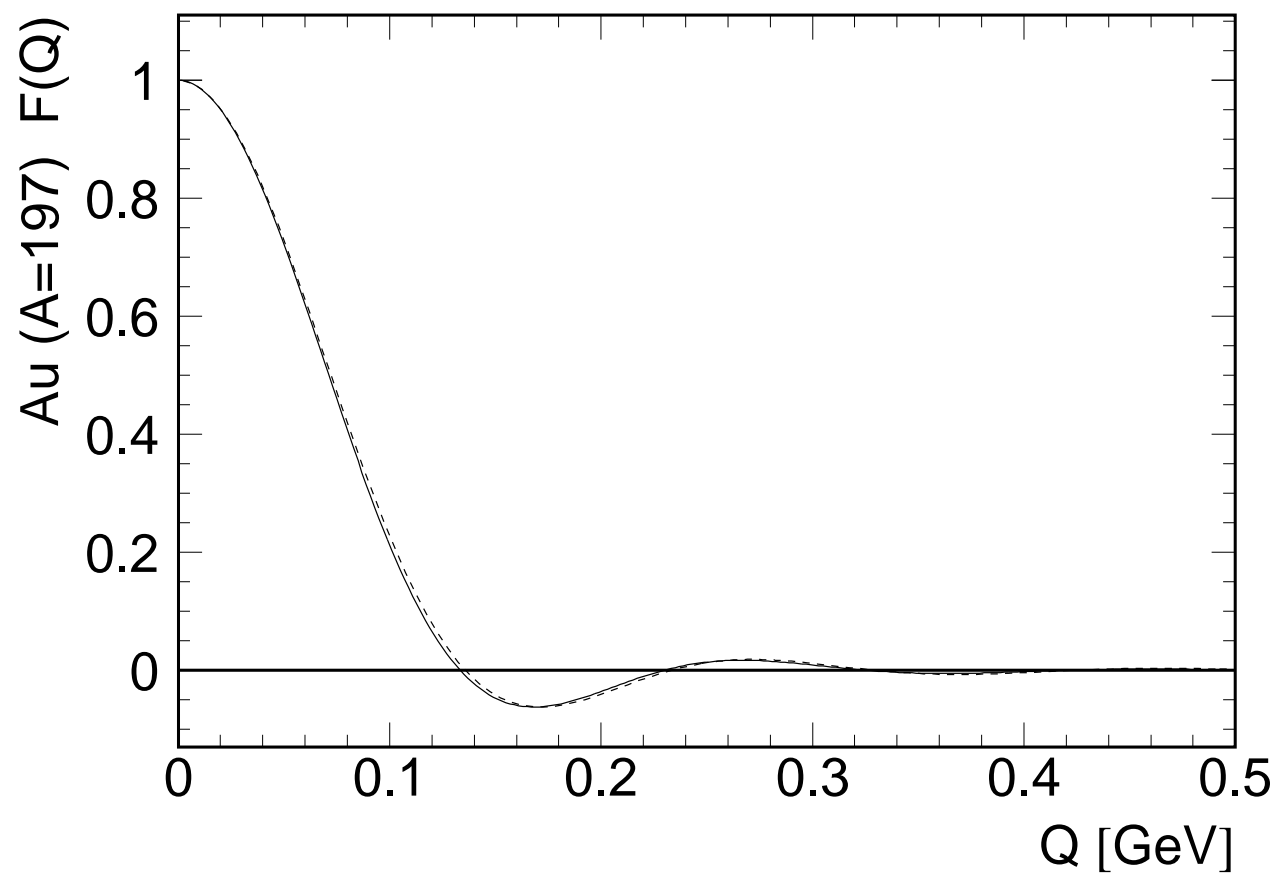

FIG. 2. The electromagnetic form factor for gold. The solid line is the exact result from Fourier transformation of a Woods-Saxon potential, and the dotted line from Eq. (14). 


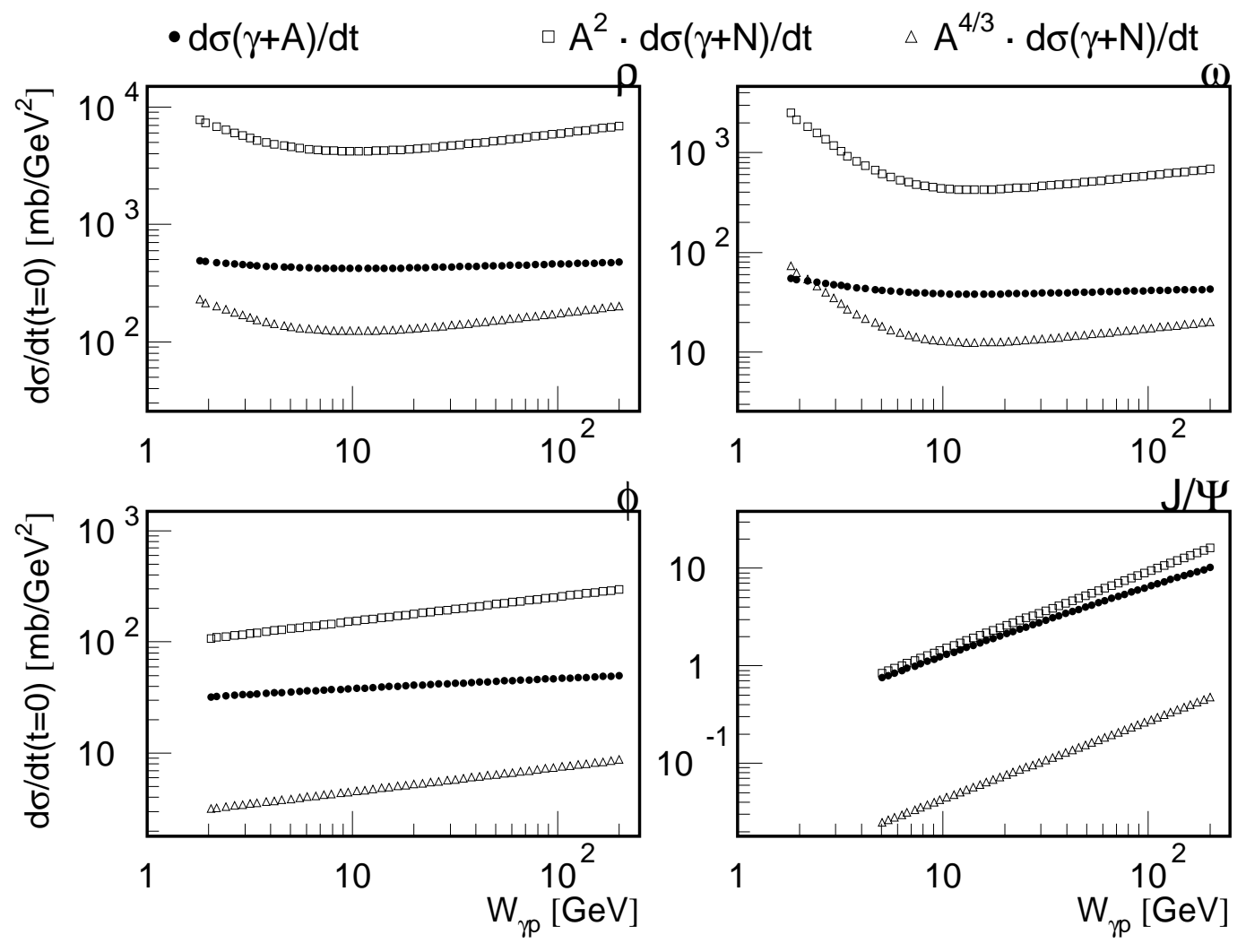

FIG. 3. $d \sigma /\left.d t\right|_{t=0}$ for coherent $\rho, \omega, \phi$ and $J / \psi$ production on a gold target. The solid circles are the Glauber calculation, while the open squares and triangles show the $A^{2}$ weak absorption and $A^{4 / 3}$ black disk scaling results respectively. The $J / \psi$ is in the weak interaction regime, while the lighter mesons are closer to the black disk. The Glauber calculation removes much of the $\gamma p$ cross section energy dependence visible with the simple scaling. 


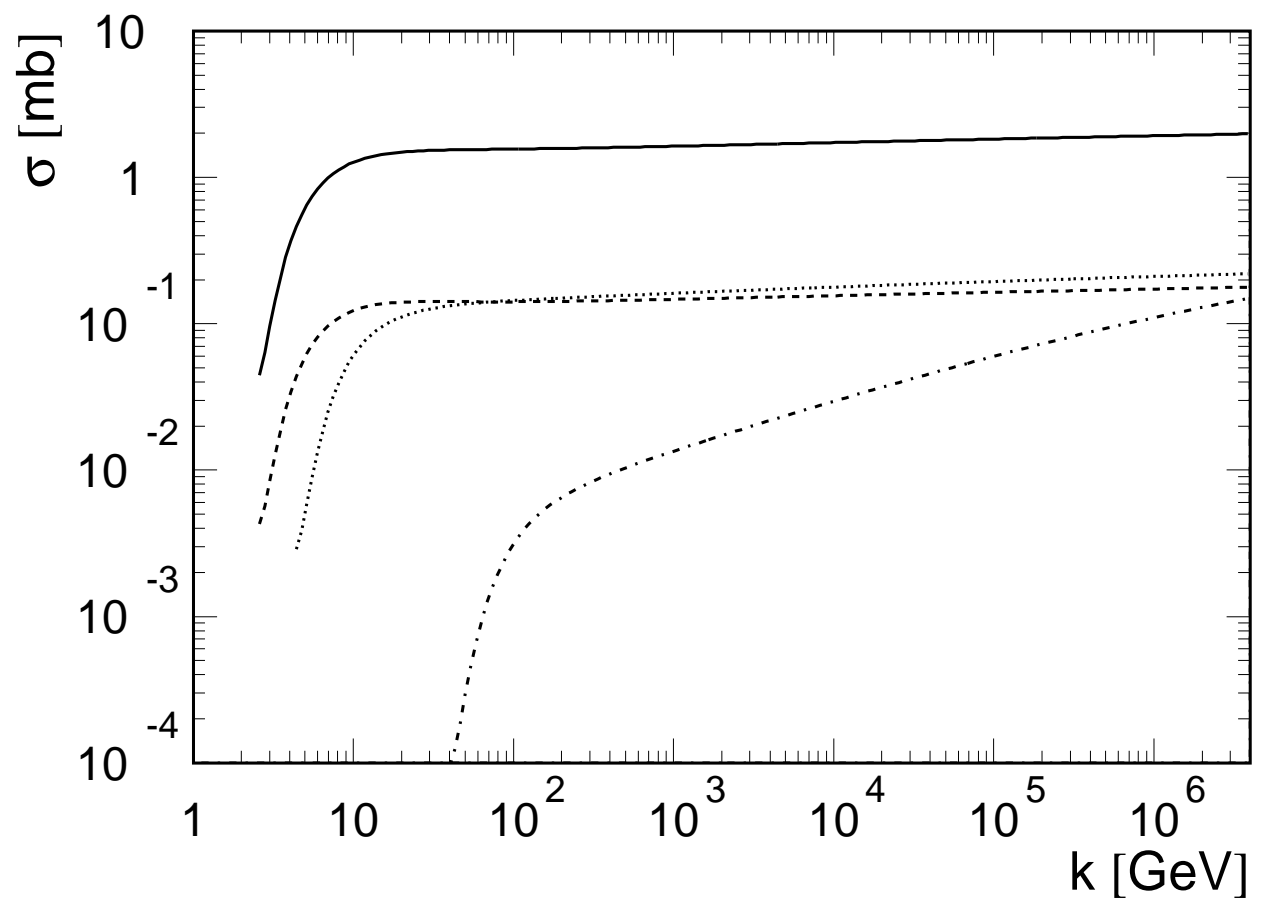

FIG. 4. Photonuclear cross sections $\sigma(\gamma A \rightarrow V A)$ for $\rho^{0}$ (solid line), $\omega$ (dashed line), $\phi$ (dotted line) and $J / \psi$ (dot-dashed line) coherent production on a gold target. 


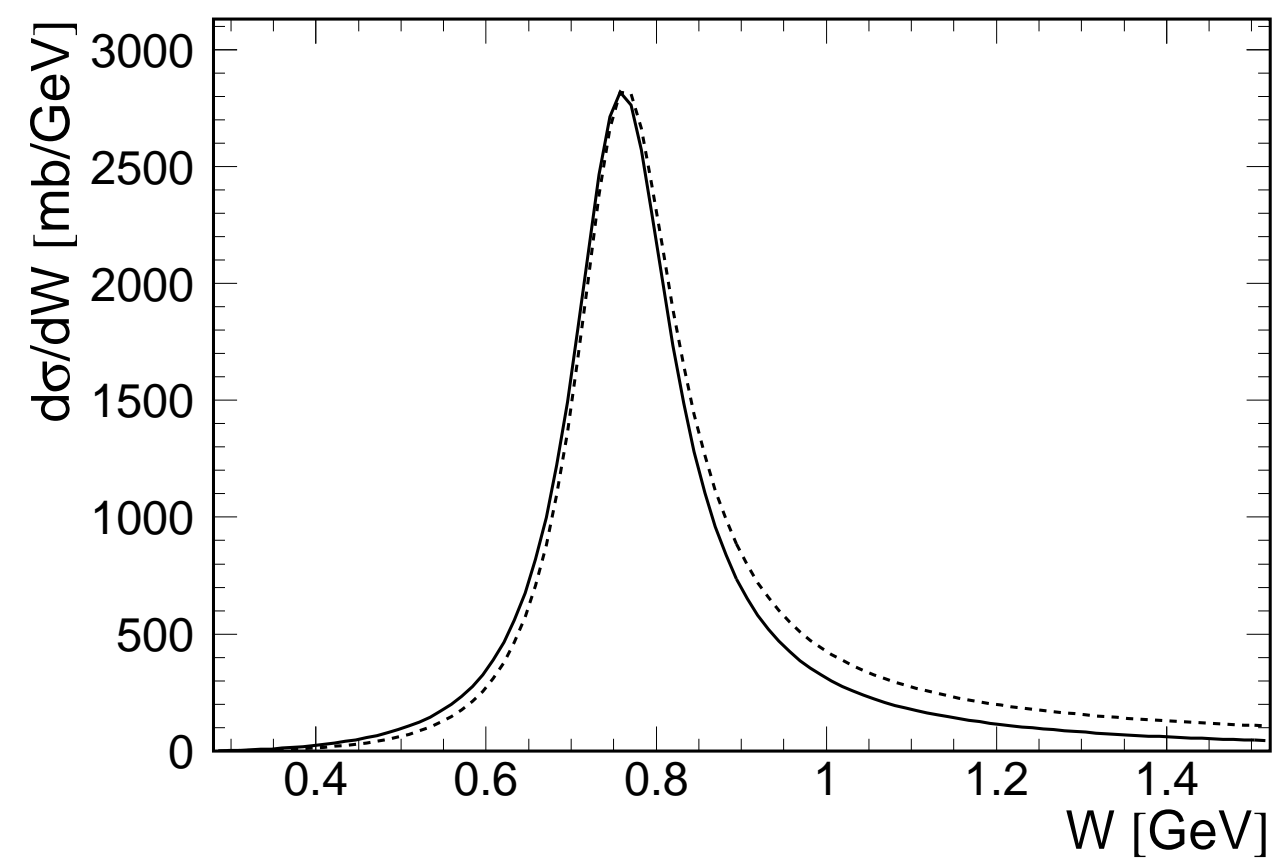

FIG. 5. Predicted $M_{\pi \pi}$ spectrum for exclusive $\rho$ photoproduction (solid line), compared with the input Breit-Wigner spectrum (dashed line). 

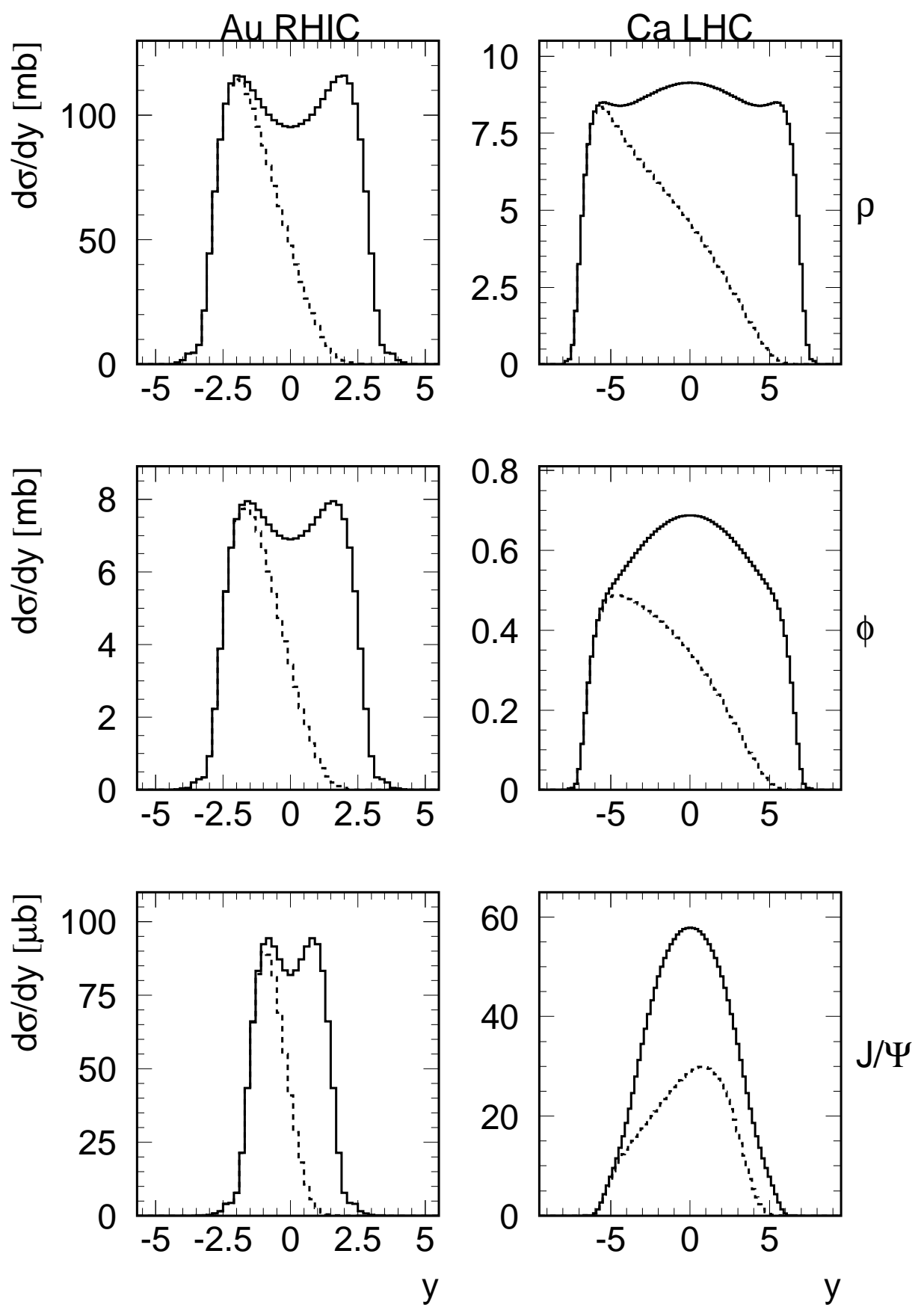

FIG. 6. The rapidity distribution of produced $\rho$ (top), $\phi$ (center) and $\mathrm{J} / \psi$ (bottom). The left panels show are for gold beams at RHIC, while the right panels are for calcium beams at LHC. The solid line is the total production, while the dashed line is for photons coming from the nucleus on the left. 

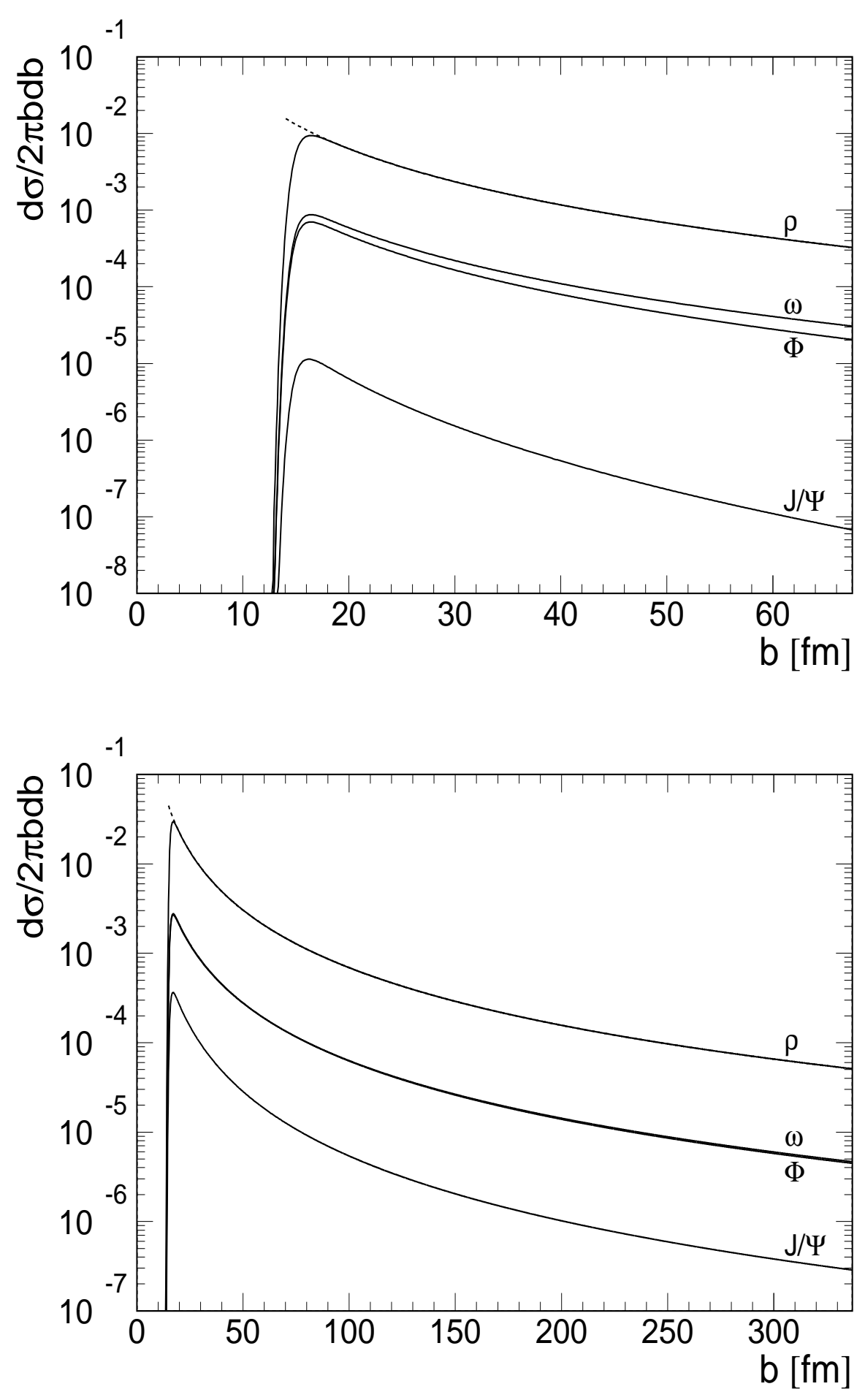

FIG. 7. Probability of vector meson production as a function of collision impact parameter for (a) gold at RHIC and (b) lead at LHC. The solid lines show the Woods-Saxon hadronic interaction probability, while the dashed lines shows the result of a hard sphere calculation for the $\rho$. 


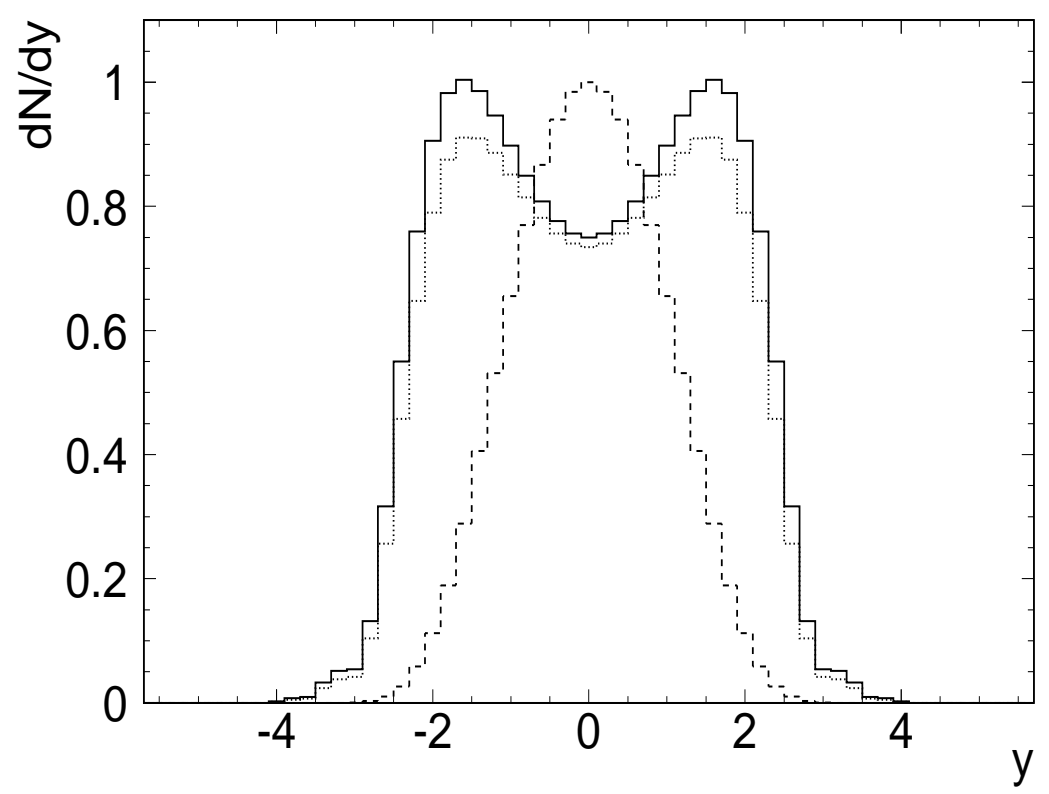

FIG. 8. Comparison of the rapidity distributions for two-photon production of the $f_{2}(1270)$ (dashed histogram) and two models of photonuclear production of a hypothetical $X(1270)$, for gold beams at RHIC. The solid histogram is for an $X(1270)$ with couplings matching the $\omega$, while the dotted histogram corresponds to Pomeron coupling only. 